\title{
Radicals of Diphenylbutadiynes and Their Interactions with Diphenylpicrylhydrazyl. A Suggestion for the Nontopochemical Polymerization Mechanism of Diacetylene
}

\author{
Gerardo Canizal, Guillermina Burillo, Jose Luis Boldu,* \\ Eduardo MuÑoz,* and Takeshi OGAWA**,† \\ Instituto de Ciencias Nucleares, Universidad Nacional Autónoma de México, Circuito Exterior, \\ Ciudad Universitaria, México DF 04510, Mexico \\ * Instituto de Física, Universidad Nacional Autónoma de México, Circuito Exterior, \\ Ciudad Universitaria, México DF 04510, Mexico \\ ** Instituto de Investigaciones en Materiales, Universidad Nacional Autónoma de México, \\ Circuito Exterior, Ciudad Universitaria, México DF 04510, Mexico
}

(Received July 18, 1995)

\begin{abstract}
Electron Spin Resonance studies of diarylbutadiynes such as diphenylbutadiyne and $p, p^{\prime}$-butadiynylenedi(methyl benzoate) have shown that these compounds contain free radicals at room temperature and that the number of radicals in the solid state increases with temperature. The color of diphenylpicrylhydrazyl (DPPH) disappears when these diacetylenes are added, indicating that the stable DPPH radicals react with the diacetylenes. The results suggest a probable initiation mechanism of the polymerization of the diacetylenes which do not undergo topochemical polymerization.

KEY WORDS Diacetylenes / Free Radicals / Electron Spin Resonance Spectra / Diphenylpicrylhydrazyl / Radical Interactions /
\end{abstract}

Conjugated diacetylenes (DAs) are interesting compounds because they undergo polymerization in the solid or molten state, giving highly conjugated polymers which exhibit third order nonlinear optical properties, and therefore there exist many studies on the subject, especially on lattice controlled topochemical polymerization. However, there has been very little research on the free radical reactivity of DAs, although their polymerization proceeds via a free radical mechanism (but is not initiated by free radicals). The present authors found that the free radical polymerization of vinyl monomers is retarded by the presence of diphenylbutadiyne (DPB $)^{1}$ and methacrylate radical interacts with DPB giving electron spin resonance (ESR) signals at its polymerization temperature. ${ }^{2-4}$ It was concluded that the retardation was not due to the chain transfer reaction but due to the interaction of DPB with the propagating radicals.

To understand the nature of such interactions between DAs and free radicals, further study with ESR spectroscopy was carried out, and a stable radical such as diphenylpicrylhydrazyl (DPPH), was mixed with DPB in order to see if it also interacts with such stable radicals. The results obtained are described in this paper.

\section{EXPERIMENTAL}

DPB and 4,4'-methoxycarbonyldiphenylbutadiyne (MCB) were prepared by the oxidative coupling reaction of phenylacetylene and $p$-ethynylmethylbenzoate, ${ }^{5}$ respectively. DPPH was supplied by Aldrich and used as received.

Titration of DPB in benzene with DPPH ethanol solution was carried out using a micro burette for the concentration ranges of $10^{-3} \mathrm{M}$ for DPB and $10^{-7} \mathrm{M}$ for DPPH. The end point was taken when the yellow

\footnotetext{
+ Author for correspondence.
}

color of DPPH solution no longer disappeared.

UV spectra were taken using a Perkin Elmer Model 553 Fast Scan UV-VIS spectrophotometer in benzene.

ESR spectra were recorded using a JEOL ESR spectrometer, Model JES-RE3X, equipped with a universal cavity, Model ES-UCX2, coupled with a temperature control system, Model ES-DVT1. Quartz tubes with 2.5 $\mathrm{mm}$ i.d. and $13 \mathrm{~cm}$ height were used for the ESR measurement. The number of radicals in the systems was calculated with the double numerical integration of the first derivative for the resonance curve from the eq 1,6

$$
A=\int_{H_{\mathrm{A}}}^{H_{\mathrm{B}}} \mathrm{d} H \int_{H_{\mathrm{A}}}^{H} \mathrm{~d} H^{\prime} S\left(H^{\prime}\right)
$$

where $H_{\mathrm{A}}$ and $H_{\mathrm{B}}$ are the initial and final parts of the resonance curve, respectively, and $S\left(H^{\prime}\right)$ is the absorption at field $H^{\prime}$. Free radical concentrations were measured by comparison with a calibrated $\mathrm{NaCl}: \mathrm{Mn}^{2+}$ crystal (calibrated by atomic absorption spectrophotometer), where both spectra were taken under the same conditions.

\section{RESULTS AND DISCUSSION}

The yellow color of the dilute ethanol solution of DPPH disappeared when DPB was added, which indicates there exist interactions of DPPH radicals with DPB. This was studied by quantitative titration of DPB benzene solution with the ethanol solution of DPPH. The results are shown in Table I. It can be seen that the DPPH consumption increased more than 20 times with temperature from 20 to $60^{\circ} \mathrm{C}$. When the colorless solution, titrated at $60^{\circ} \mathrm{C}$, was cooled to room temperature the yellow coloring of DPPH appeared again. When a yellow solution titrated with a small excess of DPPH at room temperature was left to stand for several days, 
Table I. Titration of DPB solution with DPPH

\begin{tabular}{ccccc}
\hline Run & Temp $/{ }^{\circ} \mathrm{C}$ & $\begin{array}{c}\mathrm{Mol}_{(\mathrm{DPB})} \\
\left(\times 10^{-3}\right)\end{array}$ & $\begin{array}{c}\mathrm{Mol}_{(\mathrm{DPPH})} \\
\left(\times 10^{-7}\right)\end{array}$ & $M_{(\mathrm{DPB})} / M_{(\mathrm{DPPH})}$ \\
\hline 1 & 20 & 2.42 & 1.012 & $2.39 \times 10^{4}$ \\
2 & 60 & 4.10 & 7.590 & $5.40 \times 10^{3}$ \\
\hline
\end{tabular}

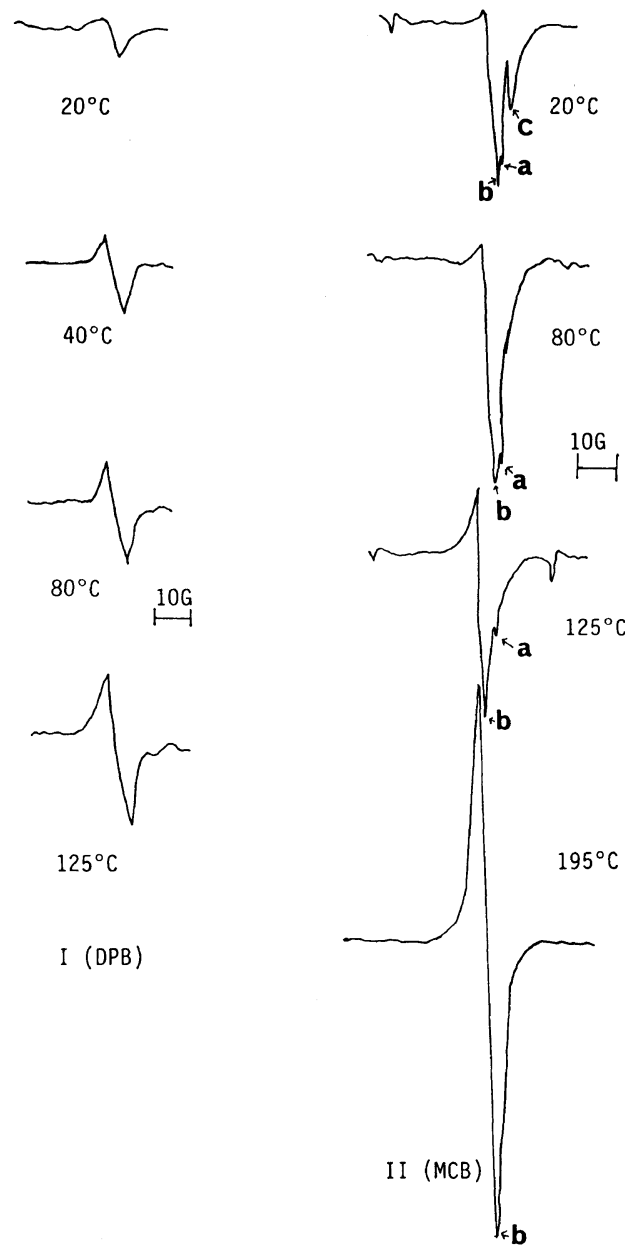

Figure 1. ESR Spectra of DPB (I) and MCB (II) taken at different temperatures. Micro wave frequency $=9.125 \mathrm{GHz}$; modulation frequency $=100 \mathrm{kHz}$; modulation amplitude $=1.00 \mathrm{G}$ (I); $0.5 \mathrm{G}$ (II except at $\left.195^{\circ} \mathrm{C}, 0.1 \mathrm{G}\right)$; receiver gain $=100(\mathrm{I}$ and $\mathrm{II}), 50\left(\mathrm{II}\right.$ at $\left.195^{\circ} \mathrm{C}\right)$; magnetic field $\left(H_{0}\right)=2350 \mathrm{G}$. the intensities of peaks due to DPPH at 274.3, 289.3, 306.4 , and $328.5 \mathrm{~nm}$ decreased slowly and the yellow color of the solution faded gradually. These observations suggest that the number of radicals increases with temperature and that the following equilibria exist:

$$
\begin{aligned}
\mathrm{DPB}(\text { or } \mathrm{MCB}) & \rightleftharpoons \mathbf{X} \\
\mathbf{X}+\mathrm{DPPH} & \rightleftharpoons \mathbf{Y}
\end{aligned}
$$

ESR spectra were taken for solid DPB and MCB at different temperatures. The results are shown in Figure 1. Unexpectedly, DPB and MCB alone showed ESR signals. The measurements were repeated after recrystallizing the diacetylenes to see if the signals are due to trace amounts of some impurities present. However, the signals were always observed, and are those of carbon radicals. Moreover it is not possible that they could have arisen from trace amounts of copper(II) salt or an oxidation product of the DAs. The signal intensity increased with temperature, suggesting more radicals are formed as the temperature rises. This temperature dependence is also indicated by the results of titration shown in Table $\mathrm{I}$. Therefore, $\mathrm{X}$ are considered as free radicals, and it is probable that DPPH interacts with them. The equilibrium shifts towards right with temperature. These results agree with the titration experiment in which coloring of DPPH reappeared when the colorless solution titrated at $60^{\circ} \mathrm{C}$ was cooled to room temperature. However, isolation of these intermediates is practically impossible due to their minute concentrations.

Analysis of the ESR signals indicates that at low temperatures there are 3 different radicals, and at higher temperatures, such as $125^{\circ} \mathrm{C}$ for $\mathrm{DPB}$ and $195^{\circ} \mathrm{C}$ for MCB at which the crystals melt, only one signal remains in both cases.

The number of the radicals of DPB and MCB in the absence and presence of DPPH at different temperatures was calculated from their respective ESR spectra, and the results are shown in Table II. MCB contains approximately fifty times more radicals than DPB. This difference may be explained by the extended conjugation of MCB by the carbonyl groups and polar effects of the carbonyl groups which decrease the electronic density of the system. Therefore, the radical of MCB is more stable

Table II. Relationship between the types of diynes, polymerization temperature, number of radicals, and concentration of DPPH

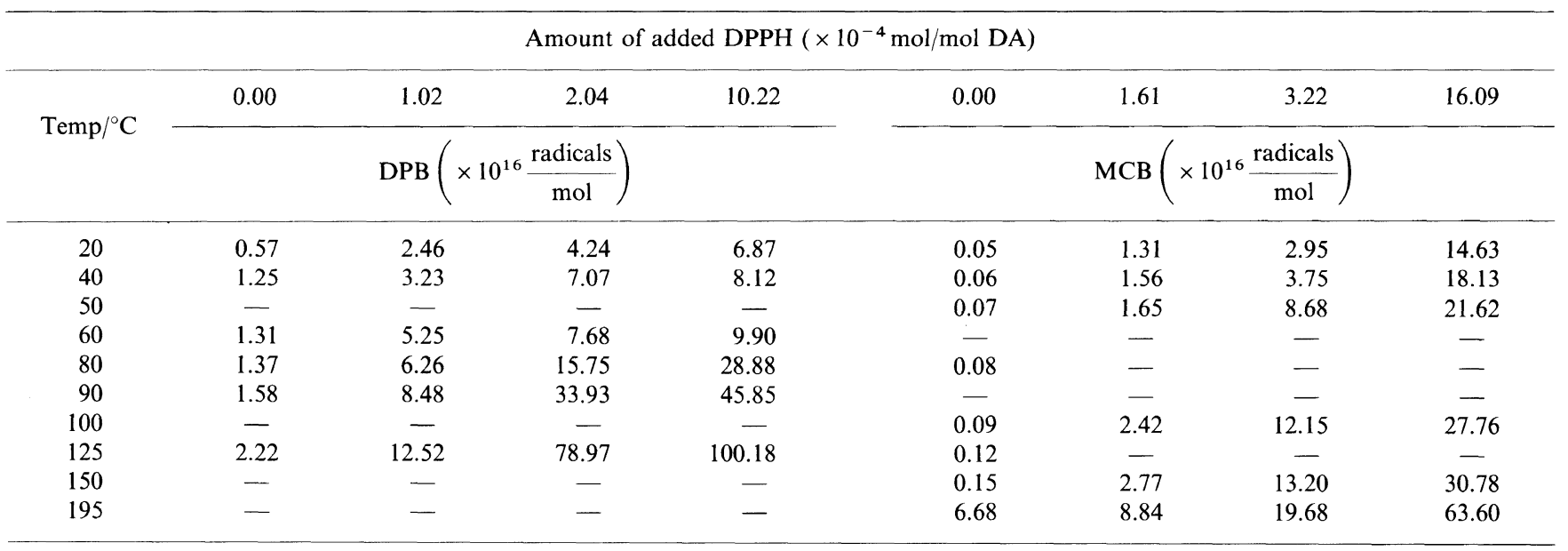


than that of DPB. The ESR spectra of MCB more clearly indicate the existence of three different radicals, as can be seen in Figure 1. It should be noted that $N, N$-diethylocta-3,5-diynylenediurethane which rapidly polymerizes by light did not give any noticeable ESR signal under the same conditions as those for DPB and MCB. The radical of the aliphatic diacetylene is probably not safficiently stable to have an equilibrium system as that shown by eq 3 . The ESR signals of those aliphatic DAs during their solid state polymerization can be detected only at low temperatures below $-78^{\circ} \mathrm{C}$. $^{7}$

Cases of topochemical polymerization of photosensitive DAs such as TS-6 (hexa-2,4-diynylenedi- $p$-toluenesulfonate), have been well studied by Sixl et al. and it is considered that a dimeric diradical is first formed, and then this initiates the polymerization. ${ }^{7}$ The butatriene structure then changes to the en-yne structure of dicarbenes when the oligomeric species are formed. In the case of DAs which do not undergo topochemical polymerization, due to a crystal packing which is unfavorable for the solid state polymerization, it is likely that the monomeric butatriene diradical is also formed, especially when substituent groups such as aryl groups, provide conjugation and thus stabilization of diradicals, but its dimerization is difficult and, if any, will be very slow because the neighboring molecule or diradical is not situated at the position for ready coupling. Therefore, the structure II may be able to form the dicarbene structure I. With increasing temperature, dimerization and oligomerization become easier thus giving the radicals of oligomeric species. The shoulder $c$ of the ESR signals in Figure 1, is assigned to the dicarbene (I), and the principal peaks $\mathbf{a}$ and $\mathbf{b}$ are assigned to the monomeric (II) and dimeric(oligomeric) (III) butatriene diradicals.

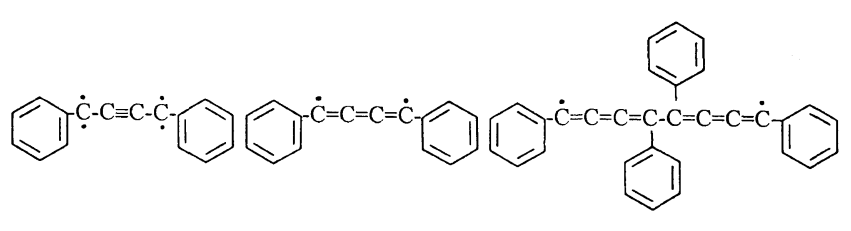

(I)

(II)

(III)

It is difficult to judge only from the results of the ESR studies of this work, if the thermal polymerization of diarylbutadiynes which do not undergo topochemical polymerization, results in 1,4-polybutadiynes as in the case of topochemical polymerization of light sensitive DAs. However, it seems that the mechanism of polymerization is probably the same in both cases, the only difference being in the crystal packing. The radicals of DAs which do not polymerize topochemically, are located far from the neighboring molecules in the crystals and they have to wait until sufficient thermal energy is provided to move around to react, or possibly they react where crystal defects shorten the intermolecular distance.

Berlin et al. earlier reported the thermal polymerization of diphenylbutadiyne at $150^{\circ} \mathrm{C} .^{8}$ Their studies mainly consisted of chemical analysis of the products obtained from the molten state polymerization. They stated that the product contains mainly the products of 1,2-polymerization. Various products are formed in this type of polymerization by the thermally induced secondary reactions among the various intermediates, and it is impossible to predict the polymerization mechanism nor the products.

Many DAs which do not undergo topochemical polymerization can be polymerized at temperatures above the melting point to give completely amorphous polymeric materials. ${ }^{9}$ Whether the polymers that are obtained consist of mainly 1,4- or 1,2-poly(DA)s is a topic of discussion. FT-IR spectra of some poly(DA)containing polyesters polymerized in the solid state, molten state and liquid crystalline state are identical. ${ }^{10}$ From the results of this work it is also thought that the 1,4-addition predominates at the initial stage of polymerization, and if temperature is too high, thermally induced random reactions take place between the 1,4structures, monomers, and other secondary products.

The interaction of DPPH radical with DPB and MCB is apparently reversible. The increase in the numbers of DA radicals with the concentration of added DPPH, as shown in Table II and Figures 2 and 3, is intriguing. The signals are not those of DPPH $(g=2.0036)$ but of DAs ( $g=2.0013$ for DPB and 2.0017 for MCB). On addition of approximately $7.5 \times 10^{-6}$ mol per mol of DPB, the DPPH signal starts to be detected, and a further increase in DPPH concentration give a strong signal. Below the above concentration of DPPH, the number of DA radicals increased considerably by the addition of DPPH.

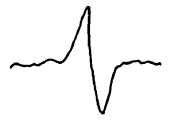

$20^{\circ} \mathrm{C}$
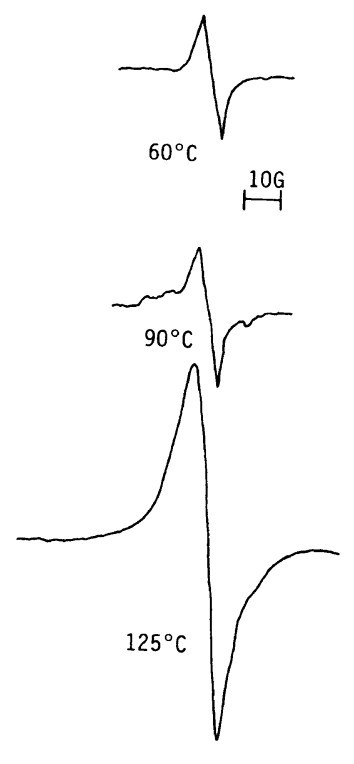
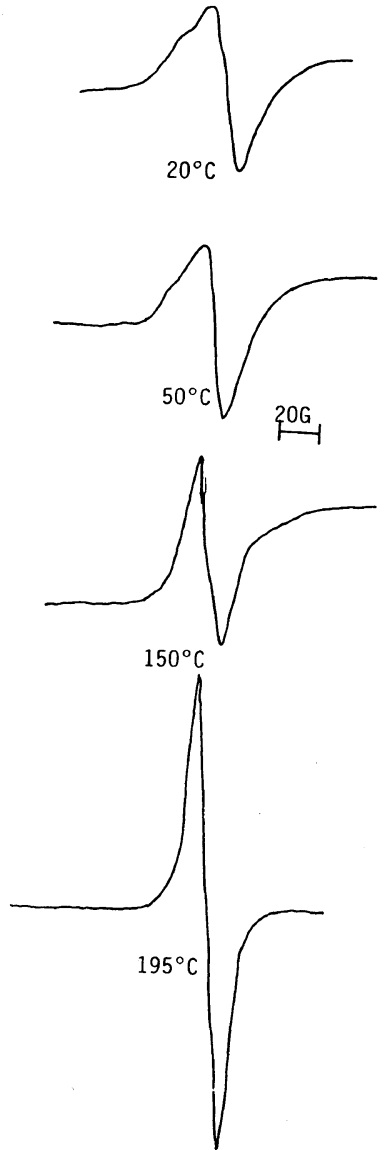

Figure 2. ESR Spectra of systems of DPPH-DPB and of DPPHMCB at different temperatures. Concentration of $\mathrm{DPPH}=2.042 \times$ $10^{-4} \mathrm{~mol} / \mathrm{mol}$ of DPB (I), $3.22 \times 10^{-4} \mathrm{~mol} / \mathrm{mol}$ of MCB(II). Microwave frequency $=9.18 \mathrm{GHz}$; modulation frequency $=100 \mathrm{KHz}$; modulation amplitude $=1.0 \mathrm{G}$; receiver gain $=100\left(\mathrm{I}: 20,60,90^{\circ} \mathrm{C}\right)$ and $50(\mathrm{I}$ : $125^{\circ} \mathrm{C}$, II: $20,50,150^{\circ} \mathrm{C}$ ), and 25 (II: $195^{\circ} \mathrm{C}$ ); magnetic field $\left(H_{0}\right)=$ $3250 \mathrm{G}$. 


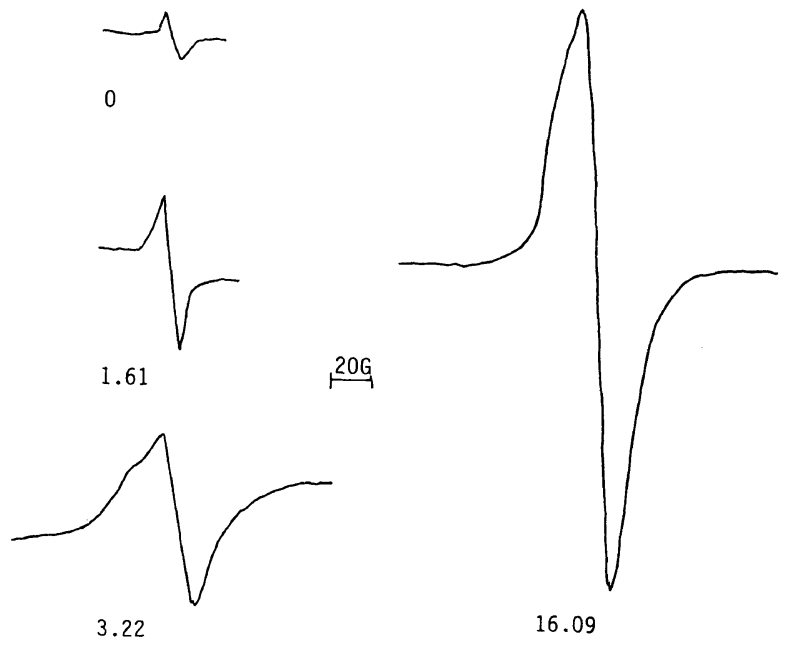

Figure 3. Effects of the concentration of DPPH on the ESR spectra of $\mathrm{MCB}$ at $20^{\circ} \mathrm{C}\left(\mathrm{DPPH} \times 10^{4} \mathrm{~mol} / \mathrm{mol}\right.$ of $\left.\mathrm{MCB}\right)$. Microwave frequency $=9.18 \mathrm{GHz}$; modulation frequency $=100 \mathrm{KHz}$; modulation amplitude $=1.0 \mathrm{G}$; receiver gain $=100(\mathrm{a}$ and $\mathrm{b}), 50(\mathrm{c})$ and 25 (d); magnetic fields $\left(H_{0}\right)=3250 \mathrm{G}$.

This suggests that DPPH has a catalytic action in the formation of DA radicals (4), which then interact with DPPH (3) as follows:

$$
\mathrm{DPPH}+\mathrm{DPB} \text { (or } \mathrm{MCB}) \longrightarrow \mathrm{X}+\mathrm{DPPH}
$$

or direct formation of interacted radical $Y$ by the DPPH radical occurs (5):

$$
\mathrm{DPPH}+\mathrm{DPB}(\text { or } \mathrm{MCB}) \rightleftharpoons \mathrm{Y}
$$

The signals of $\mathrm{X}$ and $\mathrm{Y}$ cannot be distinguished because of their very close $g$ values. In the previous study on the polymerization system of methyl methacrylate (MMA) in the presence of diarylbutadiynes, ${ }^{2-4}$ where ESR signals of polyMMA radicals stabilized by the diacetylenes appeared at the polymerization temperatures around $60^{\circ} \mathrm{C}(6)$.

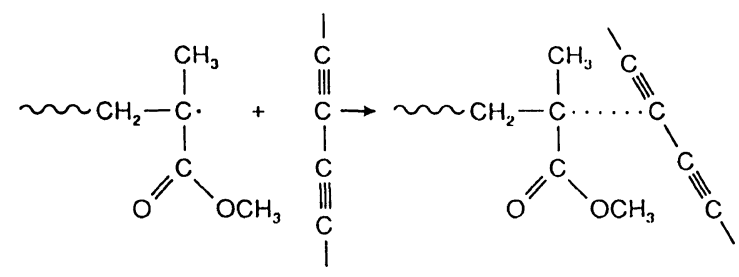

In the case of DPPH, DA radicals are probably more unstable than DPPH radicals, and DPPH seems to act as a radical stabilizer (eq 4). At the same time the radical formation of DPB and MCB is catalyzed by the stable
DPPH radicals. This hypothesis is also supported by the observation that the yellow color of the DPB solution titrated with an excess DPPH faded slowly on standing at room temperature.

Further studies on this subject are being made and the results will be reported in near future.

\section{CONCLUSION}

Some aromatic diacetylenes contain free radicals in the solid state which interact with stable radicals, such as DPPH, and also with transient radicals such as the propagating radical of poly(methyl methacrylate). ${ }^{2-4}$ Since the nonconjugated DAs such as diacetylenic diurethanes do not show any ESR signals at high temperature, the stability of these aromatic DAs is considered due to the resonance stabilization by the aromatic rings. However, because of their unfavorable crystal packing no topochemical polymerization takes place in the solid state.

More work is necessary to investigate in further details to obtain concrete information on whether DA radicals interact with other radicals, or DA interacts with the radicals. Nevertheless this work provides interesting data on the radical scavenging effect of DA molecules not only in polymerization systems but also biological and physiological systems.

Acknowledgment. The authors acknowledge DGAPA of our university for the scholarship given to G.C. for postgraduate study.

\section{REFERENCES}

1. R. E. Navarro and T. Ogawa, J. Polym. Sci., Polym. Chem., 27, 2143 (1989).

2. J. S. Huang and T. Ogawa, Polym. Bull., 23, 239 (1990).

3. G. Burillo, T. Ogawa, and J. S. Huang, J. Polym. Sci., Polym. Chem., 30, 2159 (1992).

4. G. Canizal, G. Burillo, E. Muñoz, R. Gleason, and T. Ogawa, J. Polym. Sci., Polym. Chem., 32, 3147 (1994).

5. G. Burillo, M. P. Carreón, M. E. Aguirre, and T. Ogawa, J. Appl. Polym. Sci. Polym. Symp., 49, 1 (1991).

6. T. Chang and A. H. Vahn, "Electron Paramagnetic Resonance Intensity Standard: SRM-2601; Description and Use," National Bureau of Standards Special Publication, National Bureau of Standard, Washington, D.C., 1978, Pub. No. 260-59.

7. W. Neumann and H. Sixl, Chem. Phys., 58, 303 (1981).

8. M. G. Chauser, I. D. Kalikhman, M. I. Cherkashin, and A. A. Berlin, Vysokomol. Soedin., Ser. A, 12(5), 1022 (1970); Chem. Abstr., 73, 67014h (1970).

9. T. Ogawa, Prog. Polym. Sci., 20, 943 (1995).

10. S. Fomine, S. Sanchez, and T. Ogawa, Polym. J., 27, 165 (1995) 